\title{
ÉTUDE DES ACIDES GRAS VOLATILS DU FROMAGE DE TILSIT AU COURS DE L'AFFINAGE (*)
}

\author{
par \\ J. BUDSLAWSKI et A. RÒŻOWICZ \\ Laboratoire de Chimie du Lait et des Produits laitiers à \\ l'Ecole Supérieure d'Agriculture, Olsztyn, (Pologne)
}

Parmi les produits que l'on trouve d'habitude dans les fromages au cours de leur maturation, il y a des acides gras volatils qui, dans une certaine mesure, caractérisent le goût et l'arome des fromages, mais étant en excès ils peuvent leur nuire.

Beaucoup de chercheurs ont étudié les acides gras volatils dans différentes variétés de fromages, par exemple, Zollikofer [1] dans du fromage alpestre (Bergkäse), Lehmann et Sahli [2] dans de l'Emmenthal, Ystgaard et al. [3] dans du Gouda norvégien, Scarpellino et Kosikowski [4] dans du Cheddar, Hiscox et al. [5] dans du Stilton, Long et Harper [6] dans du Provolone et du Romano, Simonart et Mayaudon [7] dans du Limburg, Sturm et Mair-Waldburg [8] dans du Romadour, Ritter et Bottazzi [9] dans du Gruyère, du Tilsit et du Limburg, Ritter [10] dans du Schabzieger et beaucoup d'autres auteurs $[11,12,13,14]$ en utilisant différentes techniques, surtout la chromatographie.

Dans ce travail, nous nous sommes proposé d'étudier qualitativement et quantitativement les acides gras volatils dans du fromage de Tilsit, sur lequel il n'y a que peu d'informations à ce sujet.

\section{Matériel expérimental et méthodes}

Nos recherches ont porté sur le fromage de Tilsit, fabriqué avec du lait pasteurisé $\left(72^{\circ} \mathrm{C}, 15 \mathrm{sec}\right.$.) et affiné durant 103 jours (13 $\pm 1^{\circ} \mathrm{C}, 85 \pm 1$ p. 100 d'humidité relative), après salage en saumure ( 4 jours, $22^{\circ}$ Bé).

Nous avons distillé les acides gras volatils du fromage avec la vapeur d'eau, selon Ritter [10], en les extrayant du distillat au moyen de n-butanol [13] et en procédant ensuite à un partage chromatographique sur papier Whatman 1, en utilisant la technique ascendante [15] dans les conditions suivantes :

- Solvant : n-butanol saturé d'eau libre de $\mathrm{CO}_{2}$;

- Révélateur : vert de bromocrézol en solution éthanolique à 0,25 p. 100 ;

(*) Cette étude fait partie du travail exécuté avec les fonds de United States Department of Agriculture, Agricultural Research Service (FGPo-151). Les auteurs expriment leur vive reconnaissance à eet organisme. 
- Eluant : eau tamponnée avec du tampon phtalo-phosphorique [16] ; température : 20 à $22^{\circ} \mathrm{C}$, temps : 20 heures ;

- Chambre chromatographique saturée des vapeurs d'éthylamine et de n-butanol.

Nous avons déterminé les acides gras volatils quantitativement par voie colorimétrique sous forme de sels d'éthylamine [11].

\section{Résultats et discussion}

Les résultats de nos recherches sont exposés dans le tableau 1. Il en ressort que le fromage de Tilsit, immédiatement après mise en moule, contient déjà une quantité importante d'acide acétique. Les six premières semaines de la maturation du fromage, il $n^{\prime} y$ avait que de l'acide acétique, et c'est seulement après 47 jours de maturation que l'on pouvait déceler des traces d'acide butyrique, et puis, successivement, les autres acides volatils libérés dans le fromage.

TABLEAU I

TENEUR DU FROMAGE DE TILSIT EN ACIDES GRAS VOLATILS AU COURS DE L'AFFINAGE

\begin{tabular}{|c|c|c|c|c|c|c|c|c|c|}
\hline & & & & $g e d u$ & fromag & ge (en j & jours) & & \\
\hline & 1 & 5 & 19 & 33 & 47 & 61 & 75 & 89 & 103 \\
\hline $\begin{array}{l}\text { Acide acétique (mg } \\
\text { p. } 100 \text { gde fromage } \\
\text { Acide propionique.. } \\
\text { Acide butyrique ... } \\
\text { Acide valérique ... } \\
\text { Acide caproique ... } \\
\text { Acide caprylique .. } \\
\text { Acidité volatile }(\mathrm{ml} \\
\mathrm{NaOH} 0,1 \mathrm{~N} \mathrm{p} . \\
100 \mathrm{~g} \text { de fromage. }\end{array}$ & $\begin{array}{r}23,6 \\
0,0 \\
0,0 \\
0,0 \\
0,0 \\
0,0\end{array}$ & $\begin{array}{r}22,8 \\
0,0 \\
0,0 \\
0,0 \\
0,0 \\
0,0\end{array}$ & $\begin{array}{r}32,4 \\
0,0 \\
0,0 \\
0,0 \\
0,0 \\
0,0\end{array}$ & $\begin{array}{r}39,8 \\
0,0 \\
0,0 \\
0,0 \\
0,0 \\
0,0 \\
\\
7,06\end{array}$ & $\begin{array}{c}52,6 \\
0,0 \\
\text { trace } \\
0,0 \\
0,0 \\
0,0\end{array}$ & $\begin{array}{r}65,2 \\
0,0 \\
0,5 \\
0,0 \\
\text { trace } \\
0,0\end{array}$ & $\begin{array}{c}67,6 \\
0,0 \\
1,0 \\
\text { trace } \\
1,2 \\
\text { trace }\end{array}$ & $\begin{array}{c}73,4 \\
0,0 \\
0,75 \\
\text { trace } \\
2,5 \\
\text { trace }\end{array}$ & $\begin{array}{c}79,6 \\
\text { trace } \\
4,5 \\
\text { trace } \\
4,0 \\
\text { trace }\end{array}$ \\
\hline Eau (en p. 100) ... & 46,69 & 42,16 & 40,96 & 40,62 & 4011 & 39,90 & 39,62 & 40,43 & 40,08 \\
\hline $\begin{array}{l}\text { Acide acétique (mg } \\
\text { p. } 100 \text { g de matière } \\
\text { sèche de fromage). } \\
\text { Acide propionique.. } \\
\text { Acide butyrique ... } \\
\text { Acide valérique ... } \\
\text { Acide caproique ... } \\
\text { Acide caprylique ... } \\
\text { Acidité volatile (ml } \\
\text { NaOH } 0,1 \text { N p. } \\
100 \mathrm{~g} \text { de matière } \\
\text { sèche de fromage. }\end{array}$ & $\begin{array}{c}44,27 \\
0,0 \\
0,0 \\
0,0 \\
0,0 \\
0,0\end{array}$ & $\begin{array}{c}38,42 \\
0,0 \\
0,0 \\
0,0 \\
0,0 \\
0,0\end{array}$ & $\begin{array}{c}54,87 \\
0,0 \\
0,0 \\
0,0 \\
0,0 \\
0,0\end{array}$ & $\begin{array}{c}67,02 \\
0,0 \\
0,0 \\
0,0 \\
0,0 \\
0,0\end{array}$ & $\begin{array}{c}87,83 \\
0,0 \\
\text { trace } \\
0,0 \\
0,0 \\
0,0\end{array}$ & $\begin{array}{c}108,5 \\
0,0 \\
0,83 \\
0,0 \\
\text { trace } \\
0,0\end{array}$ & $\begin{array}{c}111,8 \\
0,0 \\
1,66 \\
\operatorname{trace} \\
1,98 \\
\text { trace }\end{array}$ & $\begin{array}{c}123,2 \\
0,0 \\
1,24 \\
\text { trace } \\
4,19 \\
\text { trace }\end{array}$ & $\begin{array}{c}132,95 \\
\text { trace } \\
7,48 \\
\text { trace } \\
6,64 \\
\text { trace }\end{array}$ \\
\hline
\end{tabular}


Durant la maturation du fromage de Tilsit on pouvait remarquer une augmentation continue de l'acidité volatile (en ml de $\mathrm{NaOH}$ $0,1 \mathrm{~N}$ par $100 \mathrm{~g}$ de fromage) laquelle, durant les 6 premières semaines de la maturation, était causée uniquement par l'acide acétique.

Selon tous les auteurs que nous pouvons citer, l'acide acétique est toujours présent dans les fromages, mais il peut provenir soit de la fermentation lactique par la dégradation de l'acide lactique et des lactates, avec une collaboration des bactéries propioniques, soit de la fermentation hétérogène due à l'action des bactéries du groupe Coli-aerogenes. Or, l'appréciation organoleptique des fromages expérimentaux (cf. tab. 2), démontre que le processus de la maturation se poursuivait normalement. En effet, on n'avait pas remarqué dans les fromages ni de gonflement précoce, dû à l'action de colibacilles, ni de gonflement butyrique. On en peut admettre que l'acide acétique dans les fromages étudiés était un produit de la fermentation lactique, ou bien, dans le stade avancé d'affinage, il pouvait provenir, comme l'indiquent certains auteurs [17], de la dégradation et de la désamination des acides aminés qui sont toujours présents dans les fromages en maturation.

TABLEAU 2

APPRÉCIATION ORGANOLEPTIQUE DU FROMAGE DE TILSIT AFFINE

\begin{tabular}{|c|c|c|}
\hline Apparence extérieure & Apparence intérieure & Goût et odeur \\
\hline $\begin{array}{l}\text { La croûte lisse, couleur } \\
\text { jaune uniforme, talons } \\
\text { plats, arêtes arrondies. }\end{array}$ & $\begin{array}{l}\text { Ouverture normale, uni- } \\
\text { forme; couleur de la } \\
\text { pâte jaune uniforme. }\end{array}$ & $\begin{array}{l}\text { Goût et odeur purs, ty- } \\
\text { piques pour le fromage } \\
\text { de Tilsit. }\end{array}$ \\
\hline
\end{tabular}

Beaucoup de chercheurs indiquent la présence dominante de l'acide acétique dans les différentes variétés de fromages, par exemple Ritter [10] dans les fromages à pâte molle, Lehmann et Sahli [2] dans de l'Emmenthal, Ystgaard et al. [3] dans du Gouda, ete.

Après 6 semaines d'affinage du Tilsit nous y avons trouvé, à côté de l'acide acétique, les premières traces d'acide butyrique, dont la teneur dès lors augmentait continuellement pour atteindre finalement une valeur de 4,5 mg par $100 \mathrm{~g}$ de fromage. Lehmann et Sahli [2] ont trouvé aussi dans le fromage d'Emmenthal, normalement affiné, de l'acide butyrique. Dans le fromage italien Provolone, Harper [12] le trouvait déjà au premier jour de la maturation, de même Zollikofer [1], dans le fromage Alpestre âgé de 3 à 4 mois, trouvait $89 \mathrm{mg}$ d'acide butyrique par $100 \mathrm{~g}$ de fromage, la quantité dépendant d'ailleurs de la température d'affinage. 
L'acide butyrique dans le fromage peut provenir soit de la fermentation butyrique, soit de la lipolyse de la matière grasse du fromage, soit de la dégradation des protéines. Zollikofer [1] donne des indications sur la possibilité de la formation de l'acide butyrique à partir de la thréonine, méthionine ou de l'acide glutamique, et Ritter et Bottazzi [9] à partir de la valine et de la norvaline. Donc la présence d'acide butyrique dans le fromage ne résulte pas toujours de la fermentation butyrique. Puisque dans nos fromages expérimentaux on ne trouvait pas de signes de fermentation butyrique, il faut admettre que l'acide butyrique $\mathrm{y}$ était surtout de provenance lipidique ou protéique, ou de l'une et de l'autre.

Peu après l'apparition de l'acide butyrique, on trouvait aussi dans le fromage de Tilsit d'autres acides volatils, tels que l'acide eaproïque, eaprylique, valérique et enfin l'acide propionique. L'acide caprylique dans les fromages provient de la lipolyse [1], tandis que l'acide caproïque peut provenir soit de l'hydrolyse de la matière grasse du fromage, soit de la dégradation des acides aminés, aussi bien que les autres acides gras volatils à bas poids moléculaire. Sturm et Mair-Waldburg [8] indiquent bien la possibilité de formation des acides volatils à partir de la leucine ou de la valine, et Ritter et Bottazzi [9] ont montré qu'il est possible que l'acide valérique se forme à partir de la leucine et de l'isoleucine.

Dans nos fromages de Tilsit nous n'avions trouvé que des traces d'acide propionique, mais il est généralement connu que cet acide est souvent présent dans les fromages, surtout dans les fromages à pâte cuite. D'après Zollikofer [1], existe aussi la possibilité de formation d'acide propionique à partir des acides aminés tels que l'alanine, la sérine ou l'acide glutamique; mais ceci se produit à un stade avancé de la maturation des fromages, ce qui a eu lieu aussi dans notre fromage Tilsit.

\section{Conclusions}

De nos recherches ont peu conclure que l'acidité volatile du fromage de Tilsit, dans le premier stade de la maturation, est due uniquement à l'acide acétique, et, plus tard, aussi à d'autres acides gras volatils. Dans le fromage de Tilsit à affinage normal, l'acide acétique provient surtout de la fermentation lactique, et les autres acides volatils résultent soit de l'hydrolyse de la matière grasse, soit de la dégradation des acides aminés ou de ces deux sources à la fois.

L'appréciation organoleptique des fromages expérimentaux a montré une certaine corrélation entre l'intensité du goût et de l'arôme et l'augmentation de la teneur des fromages en acides gras volatils. 


\section{Summary}

The study of volatile fatty acids of ripening Tilsit cheese is presented. It follows from the obtained data that the volatile acidity of Tilsit cheese during ripening, in first six weeks, almost exclusively is due to the presence of acetic acid and only at a later stage to other volatile acids, butyric and caproic, too.

Acetic acid originates mainly from the fermentation of lactose ; butyric, caproic and caprylic acids may be develop in the lipolytic degradation of milk fat, wherease the remaining volatile fatty acids (valeric and propionic acids) probably originate from the degradation of amino acids.

During organoleptic tests with experimental cheeses a relation between the intensity of flavour and an increase in the quantity of volatile fatty acids has been found.

\section{BIBLIOGRAPHIE}

[1] E. Zollikofer. $X V^{\mathrm{e}}$ Congrès Int. de Laiterie, [2], 716, 1959.

[2] W. von Lehman et K. Sahli. XIV e Congrès Int, de Laiterie, [2/2], 314, 1956.

[3] O. M. Ystgaard, G. Syrrist et E. Brandsaeter. XVe Congrès Int. de Laiterie, [2], 893, 1959.

[4] R. Scarpelino et F. V. Kosikowski. J.Dairy Science, [45], 343, 1962.

[5] E. R. Hiscox, J. Harrison et J. Z. Wolf. J. Dairy Res., [18], 296, 1951.

[6] J. E. Long et W. J. HarPer. J. Dairy Science, [39], 245, 1956.

[7] P. Simonart et J. Mayaudon. XIVe Congrès Int. de Laiterie. [2/2], $541,1956$.

[8] W. Stínum et H. Mair-Waldburg. Milchwissenschaft, [13], 167, 1958.

[9] W. Ritter et V. Botrazzi. Schweiz. Milchztg. [82]; Wiss. Beil., no 38, 1956.

[10] W. RitTer. Milchwissenschaft, [10], 125, 1955.

[11] E. R. Hiscox et N. J. Berridge. Nature (London), [166], 522, 1950.

[12] W. J. Harper. J. Dairy Science, [36], 808, 1953.

[13] B. Lindquist, T. Störgards et Max-Britt Goransson. XIII C Congrès Int. de Laiterie, [3], 1250, 1953.

[14] S. Mattsson. Meddelende no 27. Sta. Mejeriförsök, Alnarp, 1950.

[15] M. V. Fatenva. Usp. sovr. biol., 50, 152, 1960.

[16] M. Struszynski. Analyse technique et quantitative, [1], 271, 1955. Varsovie.

[17] E. Baldwin. La biochimie dynamique, p. 319, 1959, Varsovie. 